\title{
Anti-Leishmanial Activity of Pelargonium roseum Essential Oil on Growth of Leishmania infantum Promastigotes
}

\author{
Mohaddeseh Abouhosseini Tabari \\ (PhD) \\ Faculty of Veterinary Medicine, Amol \\ University of Special Modern \\ Technologies, Amol, Iran \\ Mohammad Reza Youssefi (PhD) \\ Department of Veterinary Parasitology, \\ Babol-Branch, Islamic Azad \\ University, Babol, Iran \\ Bibi Razieh Hosseini Farash (PhD) \\ Department of Parasitology and \\ Mycology, Faculty of Medicine, \\ Mashhad University of Medical \\ Sciences, Mashhad, Iran \\ Mohammad Amin Ebrahimi (DVM) \\ Young Researcher and Elite Club, \\ Babol -Branch,Islamic Azad \\ University, Babol-Branch, Babol, Iran \\ Niki Nabavi Mousavi (DVM) \\ Young Researcher and Elite Club, \\ Babol- Branch, Islamic Azad \\ University, Babol-Branch, Babol, Iran \\ Elham Moghaddas (PhD) \\ Department of Parasitology and \\ Mycology, Faculty of Medicine, \\ Mashhad University of Medical \\ Sciences, Mashhad, Iran \\ Corresponding author: Elham \\ Moghaddas \\ Email: moghaddase@mums.ac.ir \\ Tel: +985138002401 \\ Address: Department of Parasitology \\ and Mycology, Faculty of Medicine, \\ Mashhad University of Medical \\ Sciences, Mashhad, Iran
}

Received : 31 Dec 2016

Revised: 01 Mar 2016

Accepted: 08 Mar 2016

\section{ABSTRACT}

Background and Objectives: Due to adverse effects of pentavalent antimonial treatment of visceral leishmaniasis using herbal medicines has received a lot of attention. In this study, we evaluated inhibitory activity of Pelargonium roseum essential oil on growth of Leishmania infantum promastigotes, and compared it with that of Glucantime.

Methods: Inhibitory effects of different concentrations $(1,2.5,5,10,25,50$ and 100 $\mu \mathrm{L} / \mathrm{ml}$ ) of $P$. roseum essential oil on the growth of $L$. infantum promastigotes were studied by MTT assay after 24 and 48 hours. All the data were analyzed by one-way analysis of variance. The effect of the essential oil was later compared with that of standard dose of Glucantime.

Results: After 24 hours, different concentrations of the essential oil had no significant inhibitory effect on promastigotes of $L$. infantum. After 48 hours, all concentrations except for $1 \mu \mathrm{L} / \mathrm{ml}$ showed significant anti-leishmanial activity. In addition, the lowest concentration that had significant inhibitory effect on L. infantum promastigote was $5 \mu \mathrm{L} / \mathrm{ml}$.

Conclusion: The essential oil of $P$. roseum has favorable inhibitory activity on the growth of $L$. infantum promastigotes after 48 hours. This indicates the therapeutic potential of this plant for treatment of leishmaniasis.

Keywords: Leishmania infantum, Pelargonium, Promastigote, Meglumine antimoniate.

This paper should be cited as: Abouhosseini Tabari M, Youssefi MR, Hosseini Farash BR, Ebrahimi MA, Nabavi Mousavi N, Moghaddas E, [Anti-Leishmanial Activity of Pelargonium roseum Essential Oil on Growth of Leishmania infantum Promastigotes ]. mljgoums. 2017; 11(5):36-40 


\section{INTRODUCTION}

Visceral leishmaniasis (VL) is the most severe form of leishmaniasis caused by Leishmania infantum. The annual incidence of VL is estimated between 200,000 and 400,000 worldwide (1). The disease is endemic in different parts of Iran, but around half of infections occur in northwestern Iran, especially in the districts of Meshkin-Shahr. Dogs are the main reservoir of $L$. infantum, but the infection has been also reported in cats and rodents (2-4).

In Iran, the first-line treatment for VL is chemotherapy with pentavalent antimonial compounds. Meglumine antimoniate (Glucantime, Sanofi) and sodium stibogluconate (pentostam, GSK) are the only drugs registered for treatment of leishmaniasis (5). Considering the numerous side effects of antimony therapy, many scientists seek effective alternative herbal drugs with lower toxicity and higher efficacy (6). Vomiting, weakness, myalgia, abdominal colic, diarrhea, skin rashes, hepatotoxicity, cardiotoxicity and pain during intramuscular injection have been documented as common side effects of antimonials (7).

Pelargonium roseum is a plant native to South Africa, North America and Europe, but also found in other parts of the world. Essential oil of $P$. roseum contains geranium, citronellol, alcohol, phenyl ethanol, mannitol and amyl alcohol that are thought to have antiinflammatory, analgesic, hemostatic and astringent antidiarrheal properties (6). Effect of $P$. roseum essential oil on $L$. major promastigotes, fungi and ticks has been demonstrated, but there is no report on its effect on $L$. infantum (8-10). Several studies confirmed the anthelmintic activity and therapeutic effects of this plant on gastrointestinal parasites (11). Although several studies have investigated alternative natural drugs for treatment of leishmaniasis, no study has yet evaluated the anti-leishmanial potency of Geranium (12). In the present study, we determined in vitro effects of $P$. roseum extract on the growth and viability of $L$. infantum promastigotes and compared it with that of Glucantime.

\section{MATERIAL AND METHODS}

Leaves of $P$. roseum were collected from the Avicenna Herbarium of Islamic Azad
University, Science and Research branch, Tehran, Iran (IAUH). Essential oil of the plant was prepared by hydrodistillation from fresh leaves. Gas chromatography analysis was performed to reveal its chemical constituents. The essential oil was diluted in $2.5 \%$ dimethyl sulfoxide (DMSO), and different concentrations of the essential oil were prepared. Glucantime was purchased from Sigma Chemical Co. (St Louis, Mo.). $L$. infantum (MHOM/FR/87/LEM1098) was provided by the Parasitology Department of Tehran University of Medical Sciences. Promastigotes of L. infantum (ANKT201383) were cultured in RPMI-1640 (Sigma-Aldrich) and $10 \%$ inactivated fetal bovine serum (Sigma, Cat N: F7942) in sterile condition at $25 \pm 1{ }^{\circ} \mathrm{C}$. Motility and number of promastigotes (around $1 \times 10^{6}$ parasites $/ \mathrm{mL}$ ) in the medium were checked before MTT assay, which is used to measure cell viability. To compare the effects of Geranium and Glucantime, $500 \mu \mathrm{L}$ of medium with $1 \times 10^{6}$ cells $/ \mathrm{mL}$ promastigotes was seeded into wells of a 24-well plate. Then $2.5 \mu \mathrm{L}$ of DMSO was added to all duplicate wells except for that of control and Glucantime. Then, various concentrations of Geranium (1, 2.5, 5, 10, 25, 50 and $100 \mu \mathrm{L} / \mathrm{ml}$ ) and $100 \mu \mathrm{L} / \mathrm{ml}$ of Glucantime were added to the duplicate wells. The plate was incubated at $25 \pm 1{ }^{\circ} \mathrm{C}$ for 48 hours. Primary two wells of the plate contained only $500 \mu \mathrm{L}$ of medium without parasite and DMSO as a blank control, respectively. Finally, optical density (OD) of all wells was measured at $570 \mathrm{~nm}$ by a spectrophotometer (ELISA reader) after 24 and 48 hours. All the above processes were repeated three times. Data were analyzed by one-way analysis of variance and t-test in SPSS software version 14.0 (SPSS Inc., Chicago, IL) with significance level set at 0.05 . Finally, the anti-leishmanial potency of different concentrations of the Geranium extract was compared with standard dose of Glucantime.

\section{RESULTS}

After 24 hours, Glucantime significantly affected the viability of $L$. infantum promastigotes. However, none of the concentrations of the essential oil of $P$. roseum significantly affected the viability of $P$. 
roseum after 24 hours. In addition, there was no significant difference between the inhibitory effect of concentrations of 2.5-10 $\mu \mathrm{L} / \mathrm{ml}$ and $25-100 \mu \mathrm{L} / \mathrm{ml}$ after 24 hours (Figure 1).

After 48 hours, all concentrations of the essential oil except for $1 \mu \mathrm{L} / \mathrm{ml}$ significantly affected the growth of promastigotes (Figure 2).

In addition, the lowest concentration that had significant inhibitory effect on $L$. infantum promastigote was $5 \mu \mathrm{L} / \mathrm{ml}$.

Figure 1- Effect of $P$. roseum essential oil on $L$. infantum after 24 hours compared with Glucantime and negative control

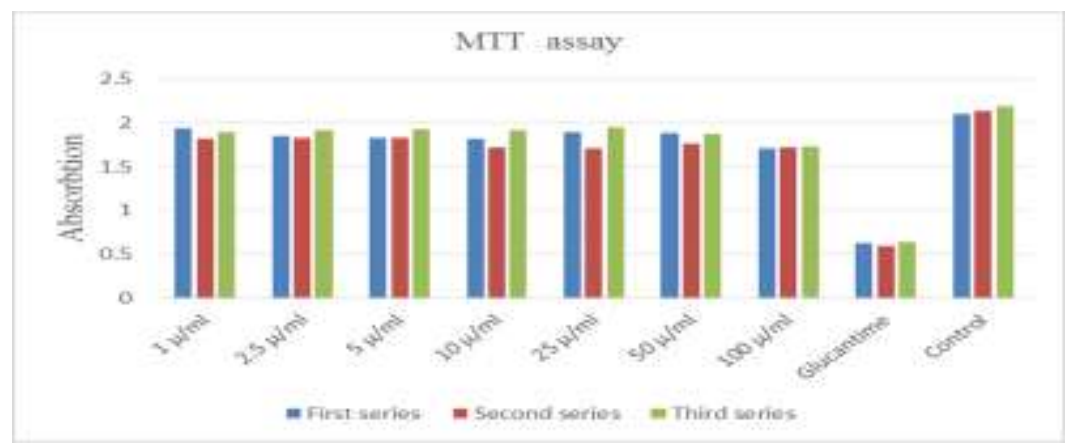

Figure 2- Effect of $P$. roseum essential oil on $L$. infantum after 48 hours compared with Glucantime and negative control

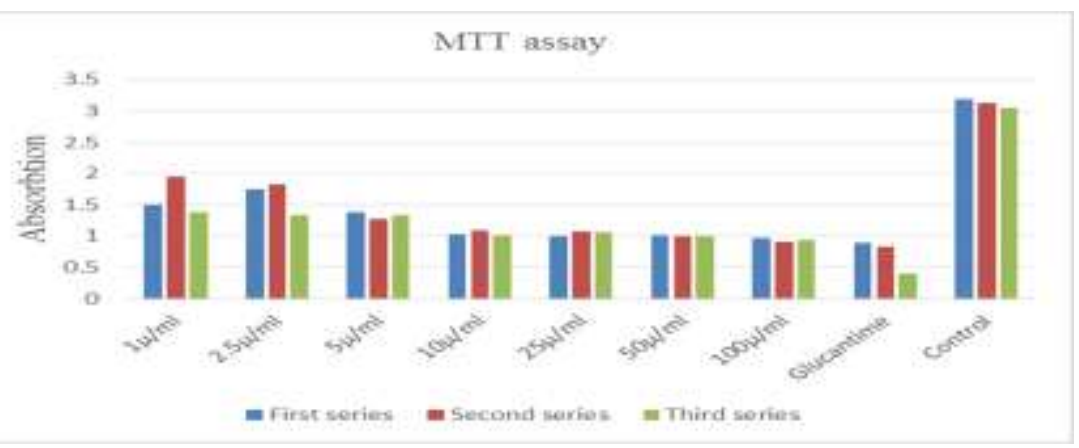

\section{DISCUSSION}

Leishmaniasis is an under-recognized disease and a major public health problem, especially in societies with poor socioeconomic status (13). High cost of treatment, resistance and side effects of drug therapy raised the need for treatment of leishmaniasis using natural herbal drugs (12).

In the present study, we determined the antileishmanial activity of Geranium extract on $L$. infantum promastigotes in coparison with Glucantime. To our knowledge, no study has yet investigated the inhibitory effects of this plant on growth of Leishmania promastigotes. According to previous studies, different plants or herbal components have inhibitory activity against various species of Leishmania (12-14).
Mansour et al. investigated the inhibitory effect of ethanolic and aqueous extracts of Vitis vinifera $L$. leaves on $L$. infantum promastigotes. They showed that the ethanolic extract is more active than the aqueous extract because it contains higher concentrations of anthocyanins, which destroys cytoplasmic and nuclear membranes of promastigotes (13). Another study showed that Thymus capitellatus have anti-parasitic activity against L. major, L. tropica and L. infantum with $50 \%$ inhibitory concentration of $35-62 \mu \mathrm{g} / \mathrm{ml}$. The extract of the mentioned plant affected mitochondrial membrane and caused cell-cycle arrest at the G0/G1 phase without causing cytotoxicity in mammalian cells (14). In the present study, all concentrations of the extract 
exhibited Glucantime-like activity against L.infantum promastigotes after 48 hours. Although the mechanism through which the Geranium essential oil affects the parasites is not fully understood, it may be due to the lipophilic properties of the extract and its percolation in cell membranes (15). Furthermore, alcoholic extract of Geranium has shown antibacterial and immune-boosting properties. The anti-inflammatory role of the extract due to presence of citronellol and geraniol, which have been shown to suppress prostaglandin E2, redness, swelling and heat $(16,17)$. Although studies showed that several essential oils from plants such as Croton cajucara, white sacaca, basil, lemongrass and Aloe vera significantly increase nitric oxide production in the leishmanicidal process, the essential oil of Geranium suppressed abnormally elevated nitric oxide. Therefore, the essential oil of Geranium could be used as a side-effect-free anti-inflammatory drug (16). Crude essential oils not only have harmful effects on parasites, but could also have adverse effects on host cells. However, we did not evaluate the toxicity of this essential oil in

\section{REFERENCES}

1. Bhattacharya SK, Dash AP. Treatment of visceral leishmaniasis: options and choice. The Lancet Infec Dis. 2016; 16(2): 142-3. doi: 10.1016/S1473-3099(15)005289.

2. Sarkari B, Naraki T, Ghatee MA, Khabisi SA, Davami MH. Visceral Leishmaniasis in Southwestern Iran: A Retrospective Clinico-Hematological Analysis of 380 Consecutive Hospitalized Cases (1999-2014). PloS one. 2016; 11(3): e0150406. doi: 10.1371/journal.pone.0150406.

3. Mohebali M, Javadian E, Yaghoobi Ershadi M, Akhavan A, Hajjaran H, Abaei M. Characterization of Leishmania infection in rodents from endemic areas of the Islamic Republic of Iran. East Mediterr Health J. 2004; 10(4-5): 591-9.

4. Sarkari B, Hatam G, Adnani S, Asgari Q. Seroprevalence of feline leishmaniasis in areas of Iran where Leishmania infantum is endemic. Ann Trop Med Parasitol. 2009; 103(3): 275-7. doi: $10.1179 / 136485909$ X398276.

5. Mohebali M. Visceral leishmaniasis in Iran: review of the epidemiological and clinical features. Iran $\mathrm{j}$ parasitol. 2013; 8(3): 348.

6. Fakhrie-Kashan Z, Arbabi M, Delavari M, TaghiZadeh M, Hooshyar H, Solaymani F. The effect of aqueous and alcoholic extracts of Pelarqonium roseum on the growth of Trichomonas vaginalis in vitro. KAUMS Journal (FEYZ). 2014; 18(4): 369-75. this study. Nevertheless, recent studies have demonstrated that essential oils of Croton argyropylloides, Artemisina annua, Menta villosa and Ligustim chuanxiong are not toxic to animal cells infected with Leishmania (12).

\section{CONCLUSION}

The essential oil of Geranium show favorable in vitro inhibitory effects against promastigotes of $L$. infantum after 48 hours. These effects are similar to that of pentavalent antimony, the most commonly used drug for the treatment of leishmaniasis. Further studies are required to confirm our results and to investigate the in vivo effects of this essential oil.

\section{ACKNOWLEDGMENTS}

The Mashhad University of Medical Sciences (Grant No. 941107) has financially supported the study.

\section{CONFLICT OF INTEREST}

The authors declare that they have no conflict of interest.

7. Frézard F, Demicheli C, Ribeiro RR. Pentavalent antimonials: new perspectives for old drugs. Molecules. 2009; 14(7): 2317- 36. doi: 10.3390/molecules14072317.

8. Hejazi H, Layeghi Ghale S, pirali kheirabadi Kh. Cytotoxic effect of essential oil of Artemisia Siberi and Pelargonium roseum on Leishmania major Promastigotes. J Shahrekord Univ Med Sci. 2015; 17(5): 13-22.

9. Carmen G, Hancu G. Antimicrobial and Antifungal Activity of Pelargonium roseum Essential Oils. Adv Pharm Bull. 2014; 4(Suppl 2): 511-514. doi: 10.5681/apb.2014.075.

10 Piralikheirabadi KH, Razzaghi-Abyaneh M, Halajian A. Acaricidal effect of Pelargonium roseum and Eucalyptus globulus essential oils against adult stage of Rhipicephalus (Boophilus) annulatus in vitro. Vet Parasitol. 2009; 162(3-4): 346-9. doi: 10.1016/j.vetpar.2009.03.015.

11. Kozan E, Akkol EK, Süntar I. Potential anthelmintic activity of Pelargonium endlicherianum. Ethnopharmacol. 2016; 187: 183- 6. doi: 10.1016/j.jep.2016.04.044.

12. Oryan A. Plant-derived compounds in treatment of leishmaniasis. Iran J Vet Res. 2015; 16 (1): 1.

13. Hotez PJ, Savioli L, Fenwick A. Neglected Tropical Diseases of the Middle East and North Africa: Review of Their Prevalence, Distribution, and Opportunities for Control. PLoS Negl Trop Dis. 2012; 6(2):e1475. doi: 10.1371/journal.pntd.0001475. 
14. Machado M, Dinis A, Santos-Rosa M, Alves V, Salgueiro L, Cavaleiro C. Activity of Thymus capitellatus volatile extract, 1, 8-cineole and borneol against Leishmania species. Vet parasitol. 2014; 200(1): 39-49.

15. Colares AV, Almeida-Souza F, Taniwaki NN, Souza CdSF, da Costa JGM, Calabrese KdS. In vitro antileishmanial activity of essential oil of Vanillosmopsis arborea (Asteraceae) baker. J Evid Based Complementary Altern Med. 2013; 4: 7-11. doi: 10.1155/2013/727042.
16. Boukhatem MN, Kameli A, Ferhat MA, Saidi F. Mekarnia M. Rose geranium essential oil as a source of new and safe anti-inflammatory drugs. Libyan $\mathrm{J}$ Med. 2013; 8: 22520. doi: 10.3402/ljm.v8i0.22520.

17. Rodrigues IA, Azevedo MM, Chaves FC, Bizzo HR, Corte-Real S, Alviano DS. In vitro cytocidal effects of the essential oil from Croton cajucara (red sacaca) and its major constituent. BMC Complementary and Alternative Medicine 2013; 13: 249. DOI: 10.1186/14726882-13-249. 